\title{
Surface roughness effect on droplet impact characterization: Experimental and theoretical study
}

\author{
J. Zohrabi Chakaneh ${ }^{1}$, S. M. Javid ${ }^{2}$ and M. Passandideh-Fard ${ }^{1 *}$ \\ ${ }^{1}$ Department of Mechanical Engineering, Ferdowsi University of Mashhad, Mashhad, Iran \\ *Email: mpfard@um.ac.ir \\ ${ }^{2}$ Department of Mechanical and Industrial Engineering, University of Toronto, \\ Toronto, Canada
}

\begin{abstract}
In this paper, the effect of surface roughness on the both normal and inclined droplet impact is investigated experimentally by image processing. The impingement of water droplets with $2.9 \mathrm{~mm}$ diameter and $1 \mathrm{~m} / \mathrm{s}$ velocity impacting on three types of stainless-steel surfaces with respective arithmetic average surface roughness values of 2.24 (Smooth), 6.04 (Medium) and $30.2 \mu \mathrm{m}$ (Rough) is examined using a high-speed camera. The dynamic behaviour of the impact including droplet deformation, the maximum spreading diameter and length, contact angle and the number of fingers is studied. Experimental results demonstrate that the maximum spreading diameter and length are lower in the surface with high roughness due to higher the kinetic energy dissipation. The results also demonstrate that surface roughness has no significant effect on contact angles. Increasing surfaces roughness not only prevent secondary droplet formation but also decrease the number of fingers formed around the droplet in the normal droplet impact. Considering the inclined droplet impact scenarios, the asymmetric spreading of droplet on inclined surfaces avoids the secondary droplet formation by decreasing the fluid kinetic energy. In the inclined impact, fingers are formed around the droplet perimeter like the normal impact. The experimental results are compared with those of the analytics available in the literature for the normal droplet impacts. Next, a simple analytical model for droplet impact on an inclined surface is developed; the predictions from this model were also compared to those of measurements. Calculated values from the analytical models agreed well with experimental data for both normal and inclined impact scenarios.
\end{abstract}

Keywords: Inclined droplet impact; roughness; maximum spreading diameter; contact angle; numbers of fingers.

\section{INTRODUCTION}

The droplet impact is an extremely active research area in the literature. It is an important phenomenon discussed in a wide spectrum of applications such as: drug delivery [1], ink-jet printing [2], combustion [3-5], wind turbines [6], aircraft icing [7], surface coating [8] and surface cooling [9]. Therefore, understanding the complicated fluid mechanic occurring during droplet/surface interaction continues to grab the attention. Many researchers have 
made numerous experimental, numerical and analytical investigations on droplet impact during the past decades $[10,11]$.

The subject of droplet impact has been studied theoretically for decades [12]. Due to the complexity of the phenomenon, much of the research on droplet impact, and especially analytical efforts, were focused on the normal impact of a droplet to a flat surface. Mundo et al. [13] expressed an empirical model describing the splashing process especially the limits of splashing in terms of Reynolds and Ohnesorge numbers by collecting data from experimental studies. An empirical model predicting the evolution of the droplet diameter during normal impact on a dry solid surface was developed by Roisman et al. [14]. Kim and Chun [15] modelled the recoiling mechanism of droplet after the impact. The effects of wetting and viscous on the recoiling mechanism were considered in their model by using modified vibrational principle [16]. Their model accurately predicted the recoiling mechanism of droplet after the impact. Roisman [17] theoretically modelled spreading film of a Newtonian fluid by using an unsteady laminar viscous flow. Their model considered the growth rate of the boundary layer thickness and closely predicts the experimental data obtained from droplet impact onto a spherical target.

The first study to examine relation between splashing and measured roughness was carried out by Stow and Hadfield [18]. They reported an empirical formula for splashing occurrence. Their experiments show that the splashing only occurred if the splashing threshold of the splash parameter was only a function of the surface roughness. The splashing threshold for rotating surfaces was reported by Mundo et al [13]. Range et al [19] correlated an experiential formula relating the critical Weber number for turning spreading to splashing.

The behaviour of the droplets impacting on a surface depends upon the impact surface conditions and the droplet properties [20]. The type of the impact surface is an important distinction made for droplet/surface interactions. The collision dynamics of the impinging droplet depends on the type of surface which can be either a solid or a liquid free surface. One of the initial studies on the effect of solid surface conditions on droplet impact was that of Chandra et al.[21]. They investigated the effect of surface temperature in droplet impacts on stainless-steel surfaces. The results showed that during the early period of impact, the droplet deformation is independent of surface temperature. Rioboo et al. [22] found that the time evolution of the droplet impact on a solid surface can be classified in six different outcomes based on influencing parameters.

The time evolution of the inclined impact of a water droplet onto a smooth optical glass surface was observed using a flash-photographic technique by Fujimoto et al. [23]. It was found that some air entrapped between the bottom of the droplet and the solid surface during impact which leads to formation of a bubble at the impact point. Li and Thoroddsen [24] obtained the time-resolved evolution of the air-layer profile during the approach of the drop and contact with the substrate. Li et al. [25] experimentally studied coalescence of a falling droplet with a stationary sessile droplet. Their experimental results demonstrated that there are three mechanism of coalescence. Kolinski et al. [26] reported that decreasing the impact velocity results in a smaller entrapped air bubble. Bouwhuis et al. [27] investigated the size of the entrapped air layer applying interferometry and found a maximum at which air compressibility can be ignored. Gier et al. [28] investigated how polymers can change the bouncing behaviour of droplets. They conducted an experiment in which an aqueous polymer solution droplets impact on a highly viscous and vertically shaken silicone oil bath. Their 
results showed that the deformation mode of the droplets significantly affects the dynamics of the bouncing.

An et al. [29] experimentally studied the maximum radius of the impacting droplet for both Newtonian and non-Newtonian liquids. Hai-Bao et al. [30] experimentally investigated the process of droplet impact on the micro-grooved surfaces. They found that the droplet impact characteristics are dependent on the surface pattern. Their results also showed that the spreading occurs in the direction parallel to the grooves. Diaz et al. [31] examined the behaviour of a gas assisted liquid droplet impacting on a solid surface through a comprehensive investigation. Their experimental results showed that gas flow leads to longer recede of droplets compared to free-falling droplets. Also, numerical results revealed that gas pressure plays an important role in the last stage of the spreading.

Based on the previous paragraphs, although many researchers have made numerous experimental, numerical and analytical investigations to completely understand the droplet impact, but the number of available studies examined effect of surface roughness on droplet impact is small. The purpose of the present study is to experimentally investigate the effect of surface roughness on both normal and inclined droplet impacts by using the image processing technique. The ImageJ software is used to measure the droplet impact characteristics (spreading factor, non-dimensional height, contact angle and number of finger). Next, an analytical expression is developed to predict spread factor for an inclined droplet impact. The model predictions are in good agreement with those of the experiments for both normal and inclined impacts.

\section{METHODS AND MATERIALS}

The experimental setup used in this study is shown in Figure 1. It consists of a droplet needle, a high-speed camera, a photoelectric sensor and a lighting system [32]. The droplet needle is capable of producing droplet sizes ranging from 1.2 to $3.2 \mathrm{~mm}$ by using a variable hypodermic needle gauge. The water droplet is allowed to grow until it separates from the needle tip and fall on the surface due to gravity. The water droplet diameter used in this study is $2.9 \mathrm{~mm}$. The impact velocity is related to the height of the free fall. The height is kept constant at $5 \mathrm{~cm}$ for all experiments performed in this study; the impact velocity, therefore, is $1 \mathrm{~m} / \mathrm{s}$ [33]. Different stainless-steel surfaces with respective arithmetic average surface roughness values of 2.24 (Smooth), 6.04 (Medium) and $30.20 \mu \mathrm{m}$ (Rough) at 0, 15 and 30 incline angles are used in the experiments. The droplet/surface interaction is captured with the use of a high-speed camera (Grasshopper, Point Grey Research) mounted with a lowdistortion telecentric microscope lens (TEC-55, Computer Company). The camera is connected to a computer with sufficient backlighting. A photoelectric sensor with a reaction time of $250 \mu \mathrm{s}$ is used to detect the droplet (ZD-L40CP, OPTEX Company). Different stages of droplet impact are photographed separately from both side view and 3D view by triggering the camera with the photoelectric sensor. The maximum spreading diameter, the maximum height, and the number of fingers is obtained through image analysis by image processing using the ImageJ software. The distance between the needle and the substrate as well as the droplet diameter are calibrated by a caliper and the image processing technique, respectively. Each experiment has been repeated four times for each time step. The uncertainty of the 
droplets size is calculated for different experiments based on the standard deviation and uncertainty analysis.

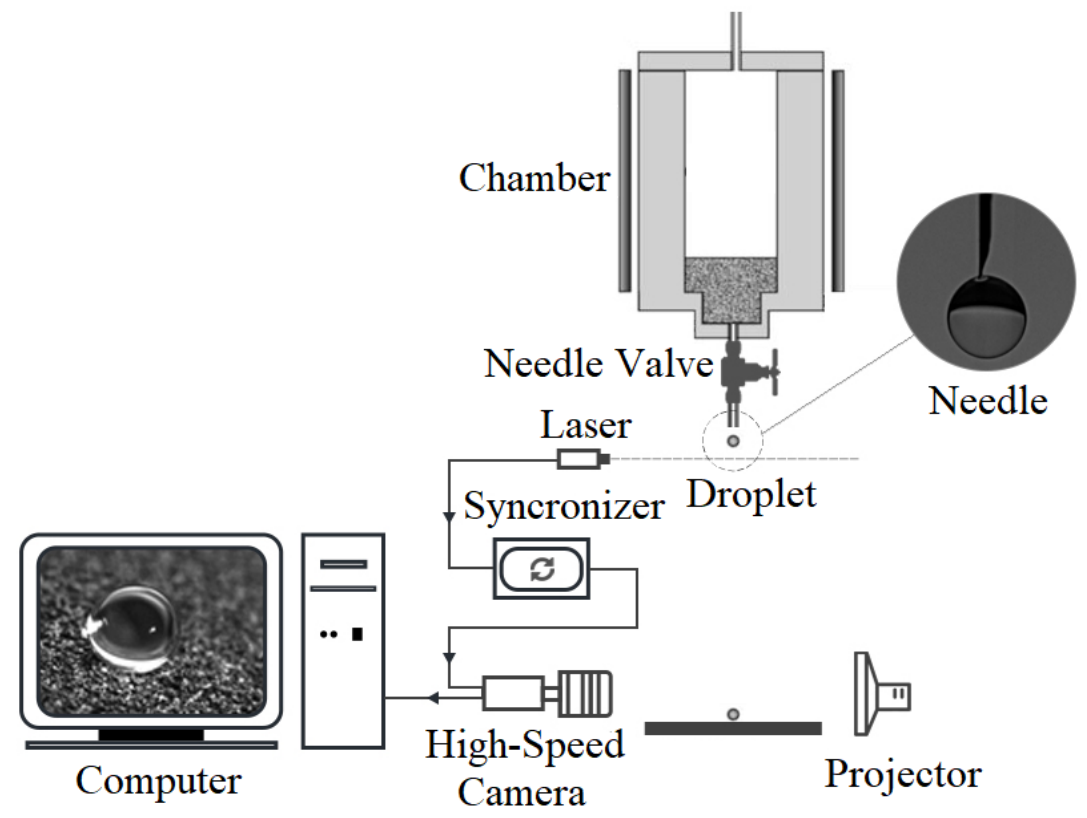

Figure 1. The experimental setup.

Table 1. Uncertainty of the droplet sizes.

\begin{tabular}{|c|c|c|c|c|}
\hline Parameter & Description & $\begin{array}{l}\text { Device/measure } \\
\text { ment technique }\end{array}$ & $\begin{array}{c}\text { Accuracy } \\
\text { (unit) }\end{array}$ & $\begin{array}{c}\text { Maximum } \\
\text { Uncertainty }\end{array}$ \\
\hline Height & $\begin{array}{l}\text { Droplet } \\
\text { maximum } \\
\text { height }\end{array}$ & $\begin{array}{c}\mathrm{CCD} \\
\text { camera/Image } \\
\text { processing }\end{array}$ & $\begin{array}{c} \pm 3 \\
(\text { pixel }) / \pm 0.06 \\
(\mathrm{~mm})\end{array}$ & $\begin{array}{c} \pm 0.0209 \\
(\mathrm{~mm})\end{array}$ \\
\hline Length & $\begin{array}{l}\text { Droplet } \\
\text { spreading } \\
\text { diameter }\end{array}$ & $\begin{array}{c}\mathrm{CCD} \\
\text { camera/Image } \\
\text { processing }\end{array}$ & $\begin{array}{c} \pm 3 \\
(\text { pixel }) / \pm 0.06 \\
(\mathrm{~mm})\end{array}$ & $\begin{array}{c} \pm 0.0323 \\
(\mathrm{~mm})\end{array}$ \\
\hline$h$ & $\begin{array}{c}\text { Distance } \\
\text { between needle } \\
\text { and surfaces }\end{array}$ & Digital Caliper & $\pm 0.01(\mathrm{~mm})$ & $\pm 0.011(\mathrm{~mm})$ \\
\hline$V$ & Impact velocity & $(V=\sqrt{2 g h})$ & $\pm 0.01(\mathrm{~m} / \mathrm{s})$ & $\pm 0.011(\mathrm{~m} / \mathrm{s})$ \\
\hline
\end{tabular}

The total uncertainty $(\delta v)$ of an experimental parameter, $v$, is related to the repetition uncertainty $\left(\partial v_{r e p}\right)$ and the equipment uncertainty $\left(\partial v_{e q p}\right)$ as follows [34]:

$$
\begin{gathered}
\delta v=\sqrt{\left(\partial v_{e q p}\right)^{2}+\left(\partial v_{r e p}\right)^{2}} \\
\partial v_{e q p}=\frac{a}{\sqrt{3}}
\end{gathered}
$$




$$
\partial v_{\text {rep }}=\frac{S}{\sqrt{n}}
$$

where , $S n$ are the half of equipment accuracy, the standard deviation, and the number of repetitions, respectively.

For a dependent parameter $\left(R=R\left(v_{1}, v_{2}, v_{3}, \ldots, v_{m}\right)\right.$, the uncertainty can be calculated as:

$$
\delta R=\sqrt{\left(\frac{\partial R}{\partial v_{1}} \cdot \delta v_{1}\right)^{2}+\left(\frac{\partial R}{\partial v_{2}} \cdot \delta v_{2}\right)^{2}+\cdots+\left(\frac{\partial R}{\partial v_{m}} \cdot \delta v_{m}\right)^{2}}
$$

In this study, the amount of uncertainty for droplet maximum height, droplet spreading diameter, distance between needle and surface and droplet impact velocity are reported in Table 1 [35].

\section{RESULTS AND DISCUSSION}

The quantitative measures of the impact are the variation of spread factor $\xi$ and dimensionless height $\eta$. The distance from the leading to the trailing edge of the droplet $(D)$ and its maximum height from the solid surface $(h)$ are measured based on which $\xi$ and $\eta$ as nondimensional factors are defined as:

$$
\begin{aligned}
\xi & =\frac{D}{D_{0}} \\
\eta & =\frac{h}{D_{0}}
\end{aligned}
$$

where $D_{0}$ shows the initial droplet diameter.

The roughness of three types of stainless-steel surfaces is expressed in non-dimensional form

$\frac{\varepsilon}{D_{o}}$, where $\varepsilon$ is the arithmetic average surface roughness. The value of $\frac{\varepsilon}{D_{o}}$ for corresponding surfaces is:

1-Smooth: $\frac{\varepsilon}{D_{o}}=\frac{2.24 \times 10^{-6} \mathrm{~m}}{2.9 \times 10^{-3} \mathrm{~m}}=0.000782$

2-Medium: $\frac{\varepsilon}{D_{o}}=\frac{6.04 \times 10^{-6} \mathrm{~m}}{2.9 \times 10^{-3} \mathrm{~m}}=0.00208$

3-Rough: $\frac{\varepsilon}{D_{o}}=\frac{30.2 \times 10^{-6} \mathrm{~m}}{2.9 \times 10^{-3} \mathrm{~m}}=0.01041$

The Weber and Reynolds numbers of impacting droplets are defined as following:

$$
\begin{aligned}
R e & =\frac{\rho V_{o} D_{o}}{\mu} \\
W e & =\frac{\rho V_{o}^{2} D_{o}}{\gamma}
\end{aligned}
$$

where $V_{o}$ is droplet velocity normal to the impact surface given by:

$$
V_{o}=U_{D} \cos (\alpha)
$$

where $\alpha$ is incline angle and $U_{D}$ is droplet velocity before impact which is $1 \mathrm{~m} / \mathrm{s}$ for all experiments. The corresponding Weber and Reynolds numbers for 0,15 and $30^{\circ}$ incline 
angles are shown in Table 2. The weber number of impacting droplets are lower that critical weber number for the splashing limit [19]. Contact angles are measured by using the ImageJ software. Measurements of most contact angles are reproducible to within of $5^{\circ}$.

Table 2. The corresponding Weber and Reynolds numbers for different incline angle

\begin{tabular}{cccc}
\hline Incline Angle & $\boldsymbol{V}_{\boldsymbol{o}}$ & $\boldsymbol{R} \boldsymbol{e}$ & $\boldsymbol{W} \boldsymbol{e}$ \\
\hline 0 & $1 \mathrm{~m} / \mathrm{s}$ & 3244 & 41.42 \\
15 & $0.966 \mathrm{~m} / \mathrm{s}$ & 3133 & 38.64 \\
30 & $0.866 \mathrm{~m} / \mathrm{s}$ & 2809 & 31.06 \\
\hline
\end{tabular}

\section{The time evolution of the normal droplet impact}

Figure 2 shows the dynamic behaviour of water droplets impacting on the horizontal stainless-steel surfaces; the corresponding time measured from the beginning of impact is given at the left of each image. According to this figure, the droplet in all cases is forced to spread out radially just after the impact. The disc-shaped spreading liquid layer is called lamella. The lamella spreads radially outward with a velocity much greater that the impact velocity [22]. The lamella deformation in time depends on the impact conditions, and inertial and capillary forces. The leading edge of the flattening droplet, known as the droplet rim, may become unstable soon after the impact. This instability may then lead to the emergence of a very regular azimuthal perturbation of the contact line [20]. The creation process of these perturbations are unknown [19]. The bump appearing on the droplet rim grow with time. The evolution of the bump radius is given by [19]:

$$
r=\left(\frac{\gamma h}{\rho}\right)^{1 / 4}\left(\theta_{o}-1 / 2 \sin \left(2 \theta_{o}\right)\right)^{-1 / 2} t^{1 / 2}
$$

where $\gamma, h, \rho$ and $\theta_{o}$ are droplet surface tension, film thickness, density and bump contact angle, respectively. Fingers are formed from the instabilities during the spreading; the size of the fingers appear to increase over time. In all cases, the fingers are slowly appearing $2 \mathrm{~ms}$ after the impact. The distinct fingers start to disappear $8 \mathrm{~ms}$ after the impact, and by $5 \mathrm{~ms}$, little evidence remains of the fingers as the fluid slowly recoils. By increasing the surface roughness, the number of fingers particularly during the early stages of the droplet spread has been decreased [19]. The surface roughness decreases the droplet kinetic energy after making contact with the surface. If the viscous dissipation caused by surface roughness is not high enough (Smooth and Medium surfaces), the droplet kinetic energy will exceed a threshold value for forming the secondary droplet. Under these conditions, capillary forces cannot maintain the integrity of the droplet and the secondary droplet may be formed (Figure 2-Smooth and Medium). In other words, if the impact surface is a relatively smooth surface, secondary droplet will be formed under the action of capillary and inertial forces. Therefore, increasing roughness not only decreases the droplet kinetic energy but also prevents secondary droplet formation. It should be noted that the lifetime of the secondary droplet in Smooth surface is bigger than that of Medium surface. Since Smooth surface has a lower viscous dissipation, its kinetic energy reduces at a lower rate during the spreading process and, therefore, the secondary droplet can bounce off the surface to a longer distance. The figures also show that the secondary droplet lifetime in Smooth surface $(22 \mathrm{~ms})$ is much larger than that of Medium surface (1ms). In Smooth surface, secondary droplet is formed $11 \mathrm{~ms}$ after the impact and then is separated from the main droplet $14 \mathrm{~ms}$ after the impact. 
For Medium surface, secondary droplet appears $11 \mathrm{~ms}$ after impact and it does not separate from the main droplet. In general, our results confirm the droplet impact stages mentioned in the literature [11, 19]. There are three main stages: the droplet flattening, the finger instability, and possible secondary droplet formation.

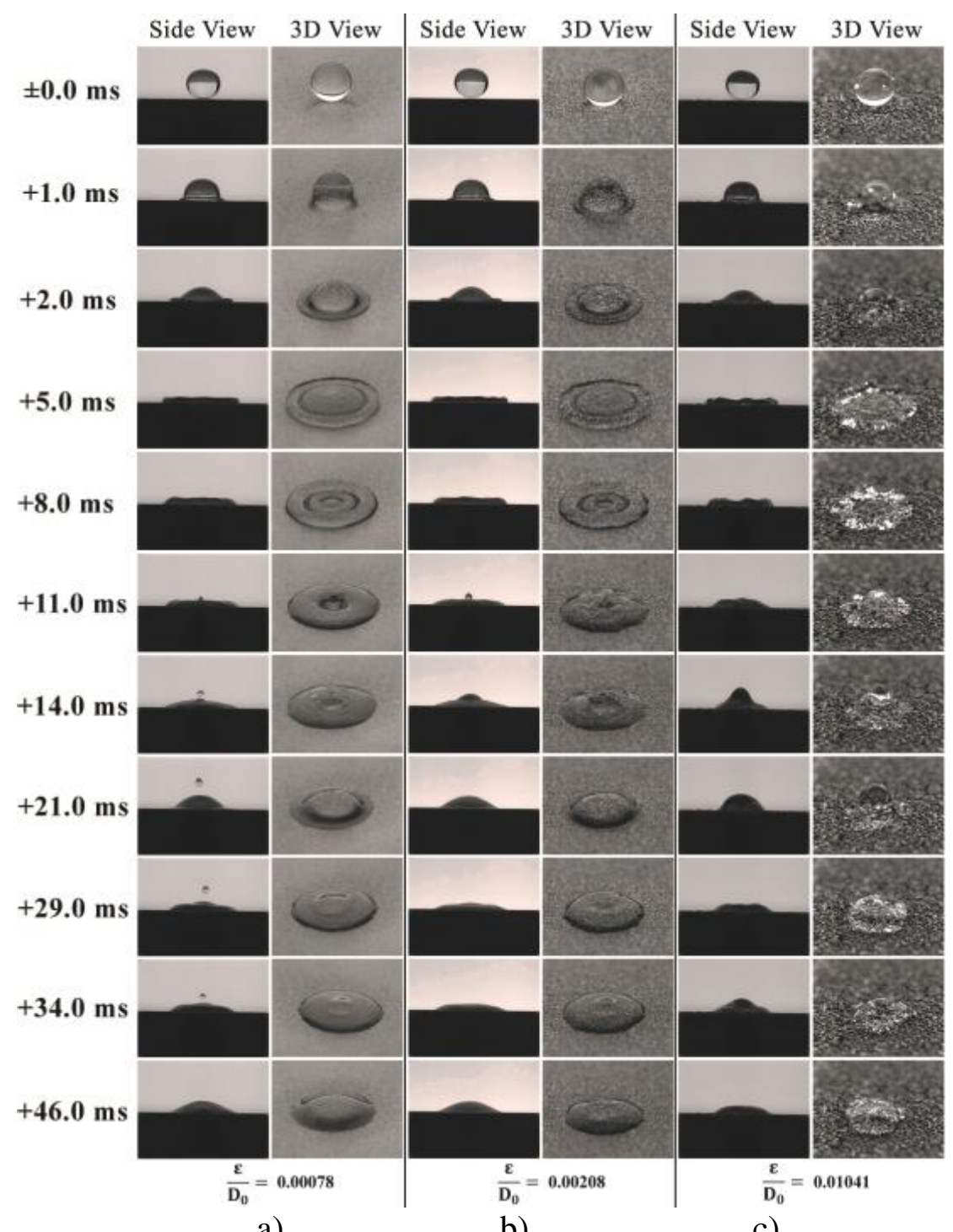

a)

b)

c)

Figure 2. The dynamic behaviour of water droplets impacting on stainless-steel surfaces with different roughness:

$$
\text { a) } \frac{\varepsilon}{D_{o}}=0.00078 \text {, b) } \frac{\varepsilon}{D_{o}}=0.00208 \text { and c) } \frac{\varepsilon}{D_{o}}=0.01041
$$

The corresponding spread factor and dimensionless height for the normal impact are shown as a function of time in Figure 3. Experimental results demonstrate that the rate of initial spreading is independent of roughness in the first 5 milliseconds after the impact. After a period of increase in $\xi$, the spread factor experiences multiple oscillations where the role 
of surface roughness on the maximum spreading diameters becomes crucial. The magnitude of oscillations for Rough surface is much higher than that of Medium and Smooth surfaces. As a matter of fact, the spread factor on Smooth and Medium surfaces do not undergo oscillations; deformation in these cases reveal a gradual flattening of the droplet. The viscous dissipation resulting from spread-recoil oscillation can significantly reduce the kinetic energy of the droplet and dampen the magnitude of spread-recoil oscillations [36]. These oscillations have more tendency to damp out quickly at Smooth and Medium surfaces. Furthermore, Rough surface resists initial spreading due to high dissipation, and these results in a smaller maximum spread factor as compared to other surfaces. The spread-recoil oscillation can be seen clearly in Figure 3(a). These oscillations exhibit a specific period and they dampen moderately through the time. It can also be observed that there is a direct relationship between dimensionless height and surface roughness; i.e. for a higher surface roughness the dimensionless height is larger. As the fingers developed in Rough surface seems to be bigger in size, the dimensionless height $\eta$ is larger.

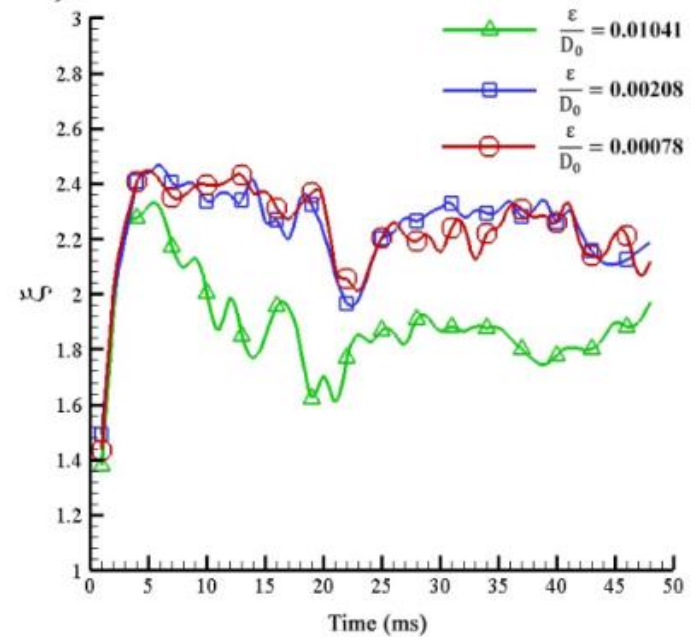

a)

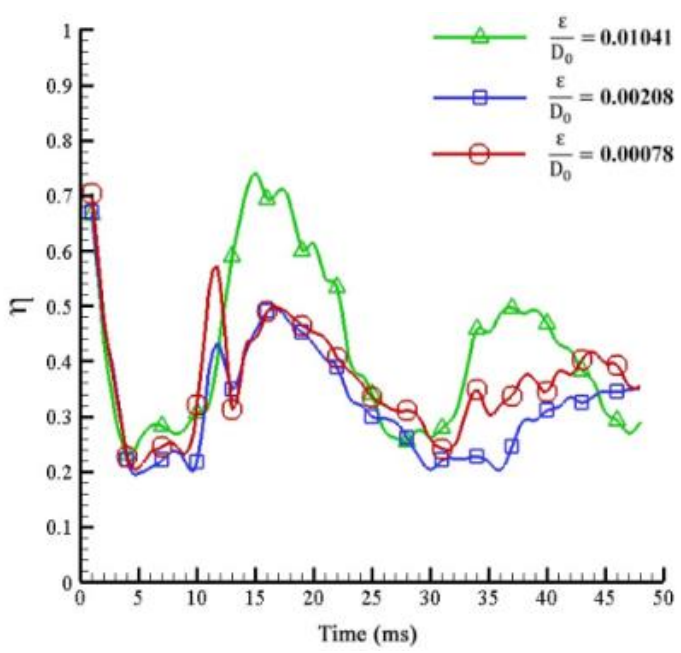

b)

Figure 3. a) The spread factor, b) The dimensionless height as a function of time for surfaces at $0^{\circ}$ incline with different roughness 


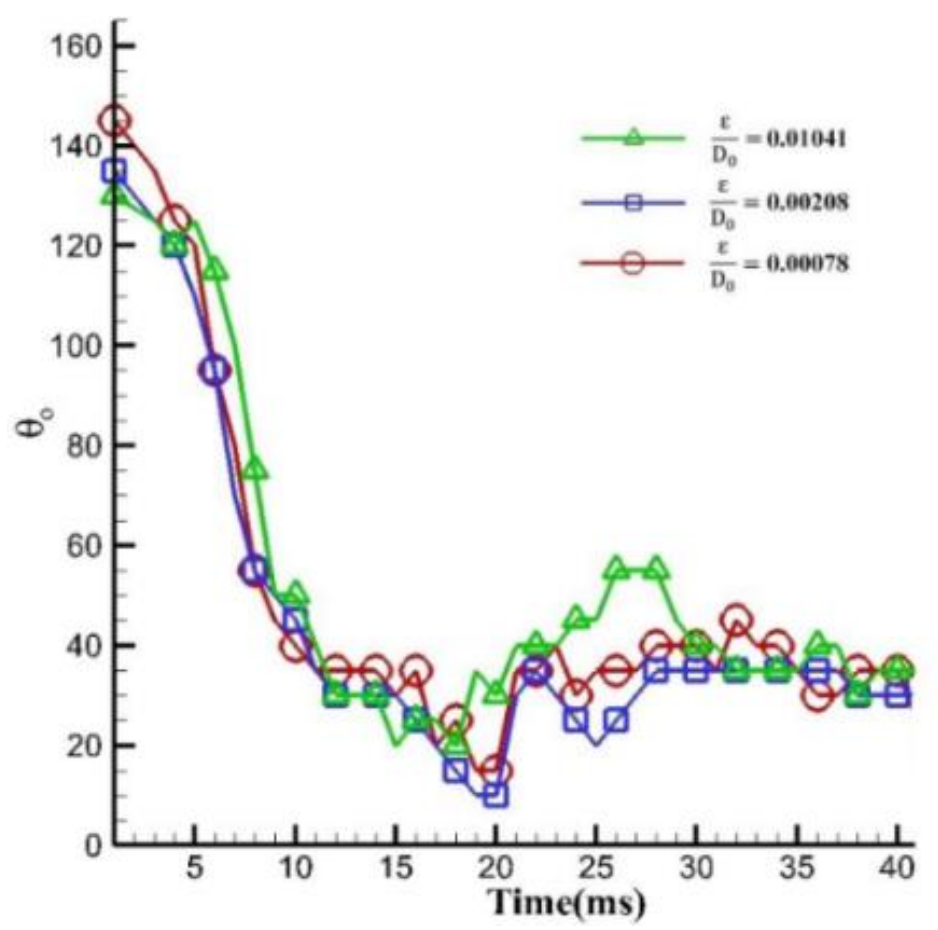

Figure 4. The contact angle variation for normal impact on stainless-steel surfaces with different roughness

In order to measure the contact angle, the ImageJ software is used. Figure 4 illustrates change of contact angle of normal impact over time for stainless-steel surfaces with different roughness. As the normal impact is symmetric, the contact angles are the same at the bottom and top of the contact line. The early behaviour for the droplets $(10 \mathrm{~ms})$ is very similar. All contact angles start with around $140^{\circ}$ value and decrease to a value of about $40^{\circ}$. After $15-20$ ms elapsed from the impact, however, a noticeable difference in contact angle can be observed between surfaces with different roughness. Also, the variation of contact angles through the time becomes more noticeable. Since the droplets impacting on Rough have a smaller maximum spread factor compared to droplets impacting on surfaces with lower roughness, their contact angle is larger than others. By $40 \mathrm{~ms}$, there is no clear difference and the contact angles become stable.

\section{Empirical formula of predicting the maximum spread factor of the normal droplet impact}

The maximum spread factor is one of the most important outcomes of a drop impacting on a solid substrate which is used in many applications [37-39]. The maximum spread factor after impact for the normal impact like Figure 5 can be expressed as follow[40]:

$$
\xi_{\max }=\frac{D_{\max }}{D_{o}}=\sqrt{\frac{W e+12}{3\left(1-\cos \left(\theta_{o}\right)\right)+4 \frac{W e}{\sqrt{R e}}}}
$$




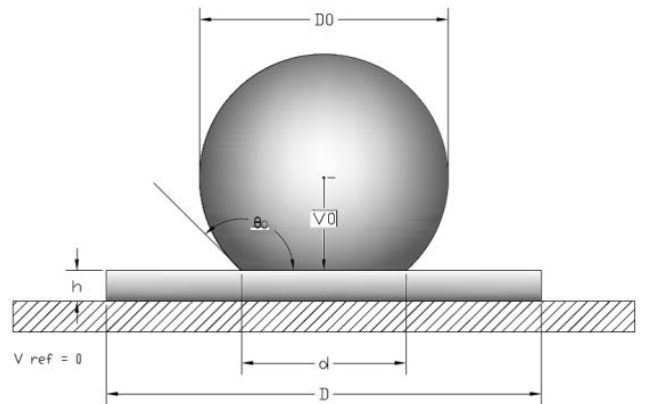

Figure 5. Model of droplet spreading in normal impact

The accuracy of experimental measurements is tested by comparison with predictions from Eq. (10) for the stainless-steel surfaces (Figure 6 and Table 3). The average discrepancy between the measured and calculated maximum spread factor for the normal impact is less than $9.1 \%$.

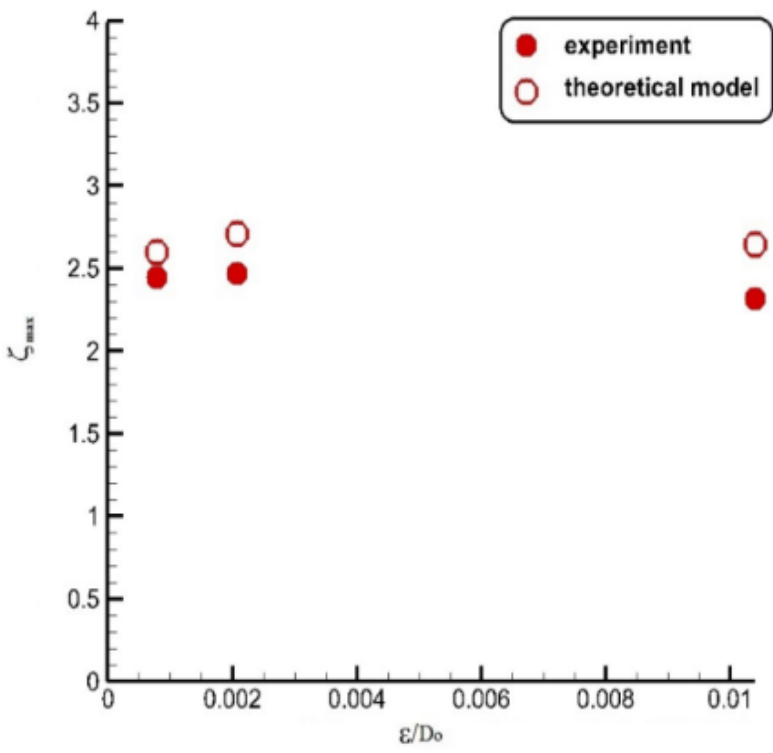

Figure 6. Comparison of maximum spread factor of theoretical model with those of experiments for the normal droplet impact

Table 3. Comparison of maximum spread factor of theoretical model with those of experiments for the normal droplet impact

\begin{tabular}{cccccc}
\hline Case & $\frac{\boldsymbol{\varepsilon}}{\boldsymbol{D}_{\boldsymbol{o}}}$ & $\boldsymbol{\theta}_{\boldsymbol{o}}$ & $\begin{array}{c}\boldsymbol{\xi}_{\text {max }} \\
\text { (theory) }\end{array}$ & $\begin{array}{c}\boldsymbol{\xi}_{\text {max }} \\
\text { (experiment) }\end{array}$ & $\begin{array}{c}\text { Difference } \\
\text { error (\%) }\end{array}$ \\
\hline 1 & 0.000782 & 131 & 2.602 & 2.448 & 5.91 \\
2 & 0.00208 & 117 & 2.710 & 2.469 & 8.89 \\
3 & 0.01041 & 125 & 2.646 & 2.320 & 12.32 \\
\hline
\end{tabular}




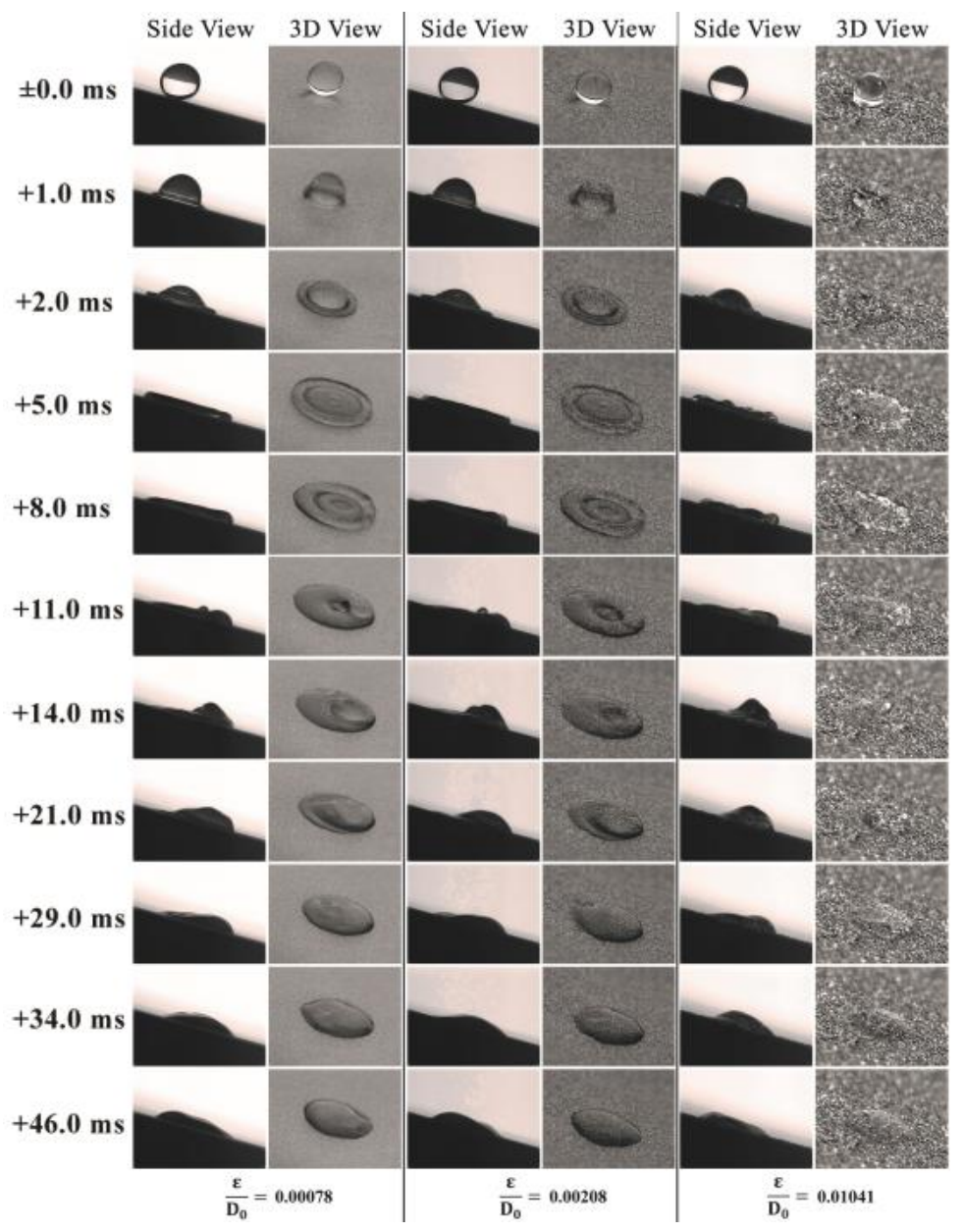

a)

b)

c)

Figure 7. The dynamic behaviour of water droplets impacting on stainless-steel surfaces at $15^{\circ}$ surface angle with different roughness:
a) $\frac{\varepsilon}{D_{o}}=0.00078$,
b) $\frac{\varepsilon}{D_{o}}=0.00208$ an
c) $\frac{\varepsilon}{D_{o}}=0.01041$

\section{The time evolution of the inclined droplet impact}

Figures 7 and 8 demonstrate the dynamic behaviour of water droplets impacting on the surfaces at 15 and $30^{\circ}$ incline angles. It can be observed that the droplet in all cases spreads out asymmetrically just after the impact which make the lamella layer development more complicated than the normal impact [41]. In the inclined impact, fingers are formed around the droplet perimeter like the normal impact. As can be seen in Figures 7 and 8, the number of fingers in inclined impact has decreased compared to normal impact. As far as the surface at $15^{\circ}$ incline angle is concerned, the fingers are slowly appearing $2 \mathrm{~ms}$ after the impact. The distinct fingers start to disappear $8 \mathrm{~ms}$ after the impact, and by $5 \mathrm{~ms}$, there is no evidence of the fingers. The time evolution of droplet impact on surface at $30^{\circ}$ incline angle is the same as $15^{\circ}$ incline angle. For a certain surface roughness, the only difference between impacts onto surfaces with different incline angle is the number of fingers. The higher incline angle results in a lower number of fingers. The asymmetric spreading of droplet impacting on 
inclined surfaces avoids forming the secondary droplet by decreasing the droplet kinetic energy. In other words, the droplet kinetic energy won't exceed a threshold value for forming the secondary droplet under these conditions.

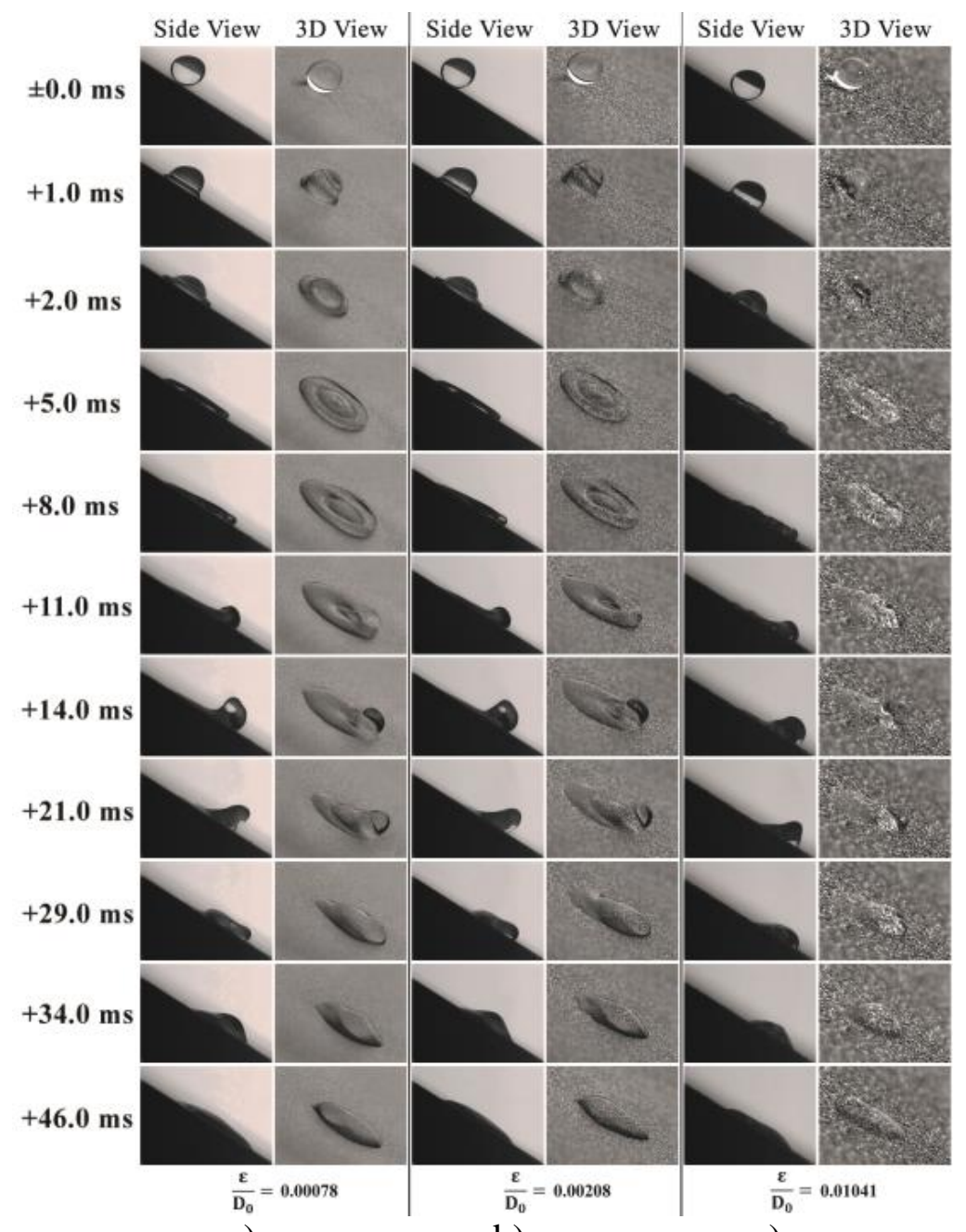

a)

b)

c)

Figure 8. The dynamic behaviour of water droplets impacting on stainless-steel surfaces at $30^{\circ}$ surface angle with different roughness:

$$
\text { a) } \frac{\varepsilon}{D_{o}}=0.00078 \text {, b) } \frac{\varepsilon}{D_{o}}=0.00208 \text { and c) } \frac{\varepsilon}{D_{o}}=0.01041
$$

Figures 9 and 10 show how spread factor and the dimensionless height change with time respectively for the surfaces at 15 and $30^{\circ}$ incline angle. As it was expected, the spread factor, $\xi$, sharply increases in few milliseconds after the impact. During this period, there is no difference in the value of factor among the surface with different roughness. The influence of surface roughness on the spread factor appears to be more significant through the time when $\xi$ starts multiple oscillations. As a matter of fact, the results show that the inclined droplet impact is sensitive to the surface roughness values during this time like the normal impact. The oscillations continue until the droplet kinetic energy is dissipated. As can be seen 
in Figures 9-a and 10-a, there is a direct relationship between viscous dissipation and surface roughness; i.e. for a higher surface roughness the spread factor is lower which means the viscous dissipation is larger. Unlike the horizontal surface, the droplet impact on the inclined surfaces spreads out asymmetrically due to gravity. The asymmetric spread leads to higher $\eta$ of the inclined droplet impact compared to the normal one. In other words, for a higher surface incline angle the $\eta$ is larger. It also can be observed that the specific period of spreadrecoil oscillations dissipating the kinetic energy does not change with increasing the surface incline angle.

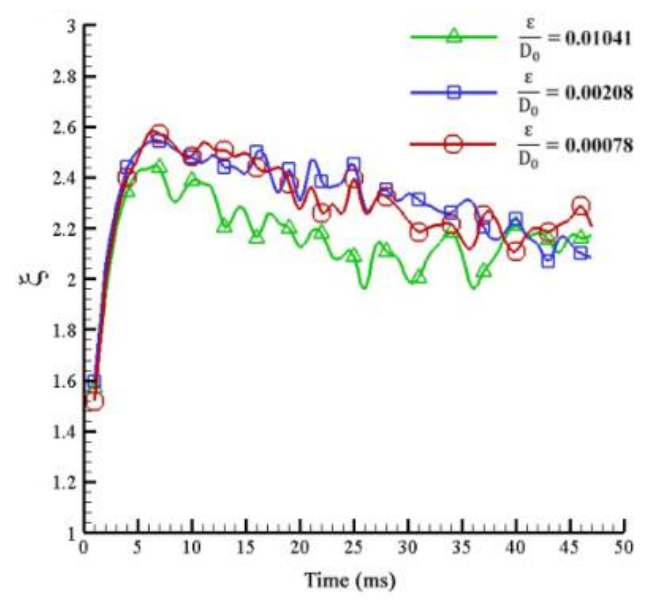

a)

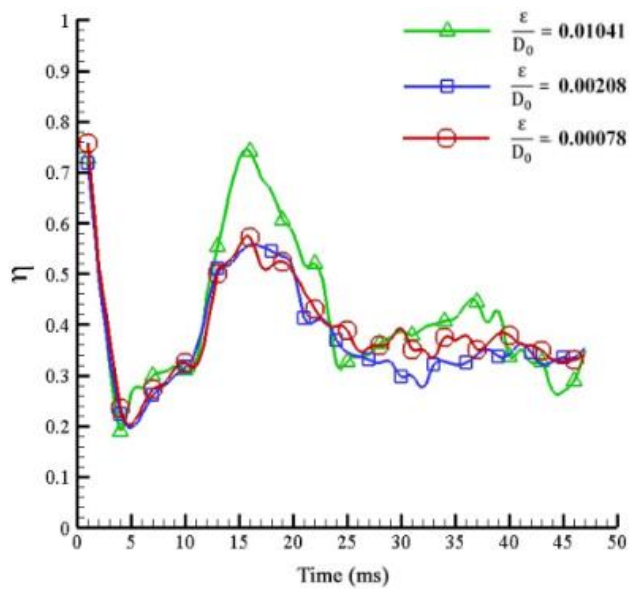

b)

Figure 9. a) The spread factor, b) The dimensionless height as a function of time for surfaces at $15^{\circ}$ surface angle with different roughness

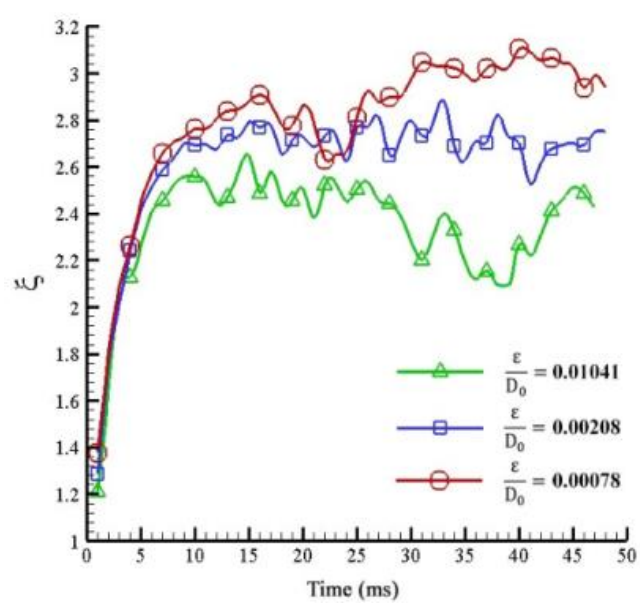

a)

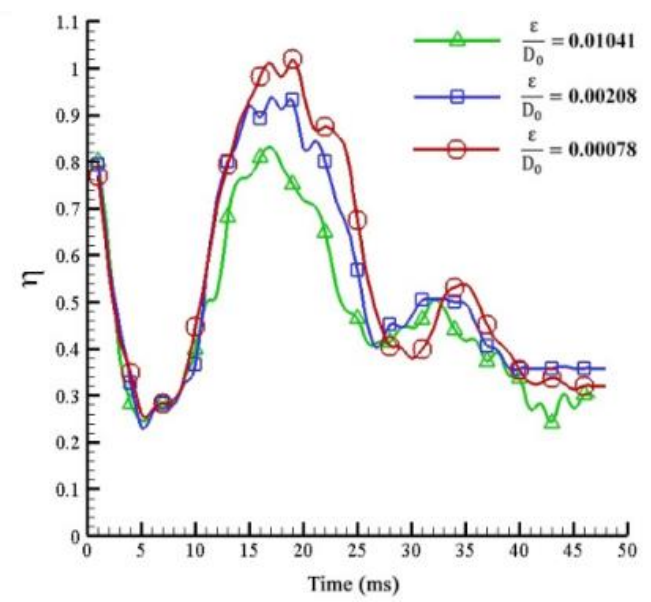

b)

Figure 10. a) The spread factor, b) The dimensionless height as a function of time for surfaces at $30^{\circ}$ surface angle with different roughness

Due to asymmetric spread of inclined impact, there are two contact angles. The contact angles are those at the bottom and top of the contact line, designated the "advancing" and "receding" contact angles, $\theta_{a}$ and $\theta_{r}$ respectively (Figure 11). 


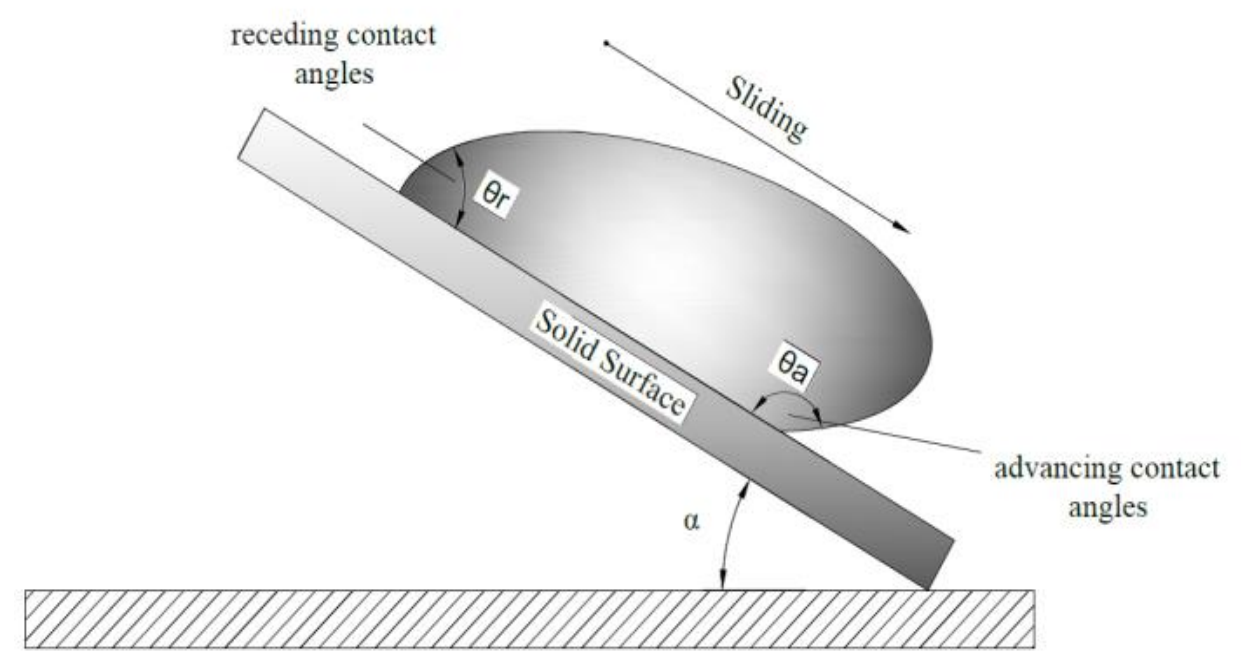

Figure 11. The inclined impact contact angles

The relation between three contact angles of equilibrium, $\boldsymbol{\theta}_{\boldsymbol{o}}$, advancing, $\boldsymbol{\theta}_{\boldsymbol{a}}$, and receding, $\boldsymbol{\theta}_{\boldsymbol{r}}$, is given as [42]:

$$
\theta_{o}=\arccos \left(\frac{C_{a} \cos \left(\theta_{a}\right)+C_{r} \cos \left(\theta_{r}\right)}{C_{a}+C_{r}}\right)
$$

where

$$
\begin{gathered}
C_{a}=\sqrt[3]{\frac{\sin ^{3}\left(\theta_{a}\right)}{2-3 \cos \left(\theta_{a}\right)+\cos ^{3}\left(\theta_{a}\right)}} \\
C_{r}=\sqrt[3]{\frac{\sin ^{3}\left(\theta_{r}\right)}{2-3 \cos \left(\theta_{r}\right)+\cos ^{3}\left(\theta_{r}\right)}}
\end{gathered}
$$

Figures 12 and 13 illustrate the temporal variation of these two contact angles. These figures reveal that $\theta_{a}$ and $\theta_{r}$, in general, are not strong functions of surface roughness. The early behaviour of the inclined impact is the same as the normal impact. There is no considerable difference between $\theta_{a}$ and $\theta_{r}$ for droplets impacting on surfaces with different roughness at all incline angles in the first $5 \mathrm{~ms}$. As can be seen, the symmetry is short-lived, however, and by $10-15 \mathrm{~ms}, \theta_{a}$ is much larger than $\theta_{r}$. From $15 \mathrm{~ms}$ to $30 \mathrm{~ms}, \theta_{r}$ stays almost constant at around $20^{\circ}$ and $\theta_{a}$ decreases gradually from about $80^{\circ}$ to $40^{\circ}$ for the impact at $15^{\circ}$ inclined angle. This trend, however, is different for the impact at $30^{\circ}$ inclined angle. Figure 13 shows that $\theta_{r}$ stays nearly steady at round $10^{\circ}$ and $\theta_{a}$ experiences a period of fluctuation and then reduces sharply from about $130^{\circ}$ to near $40^{\circ}$. The different behaviour of $\theta_{a}$ in the above-referred inclined surfaces results from the kinetic energy variation with the inclined angle. In the surface at $30^{\circ}$ inclined angle, the kinetic energy of the advancing side is high enough to keep $\theta_{a}$ nearly constant (Figure 13). However, $\theta_{a}$ reduces sharply by decreasing the kinetic energy due to viscous dissipation over time. Considering the surface at $15^{\circ}$ inclined angle, the kinetic energy of the advancing side is not as high as that of the surface at 
$30^{\circ}$ inclined angle and this results in gradual decline of $\theta_{a}$ by time (Figure 12). By $30 \mathrm{~ms}$ elapsed from the impact, much of the liquid has accumulated near the leading edge. At 40 ms, Figures 12 and 13 show less oscillations in both advancing and receding contact angles at all inclined angles.

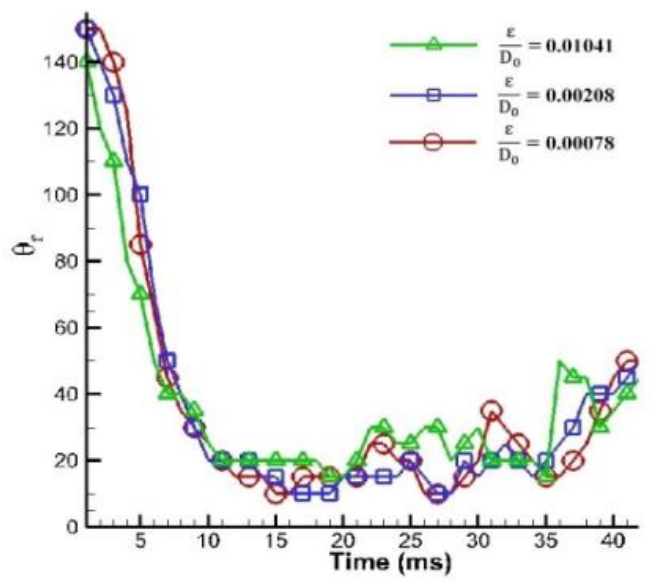

a)

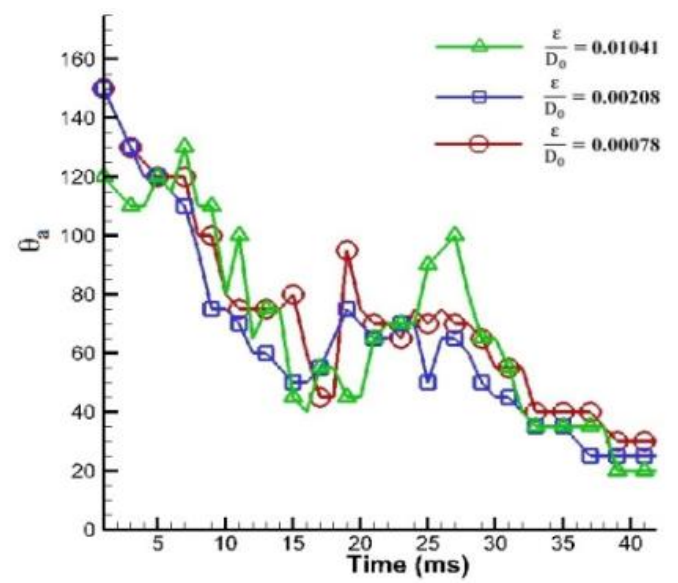

b)

Figure 12. The contact angle variation for inclined impact at $15^{\circ}$ surface angle on stainlesssteel surfaces with different roughness a) $\theta_{r}$ b) $\theta_{a}$

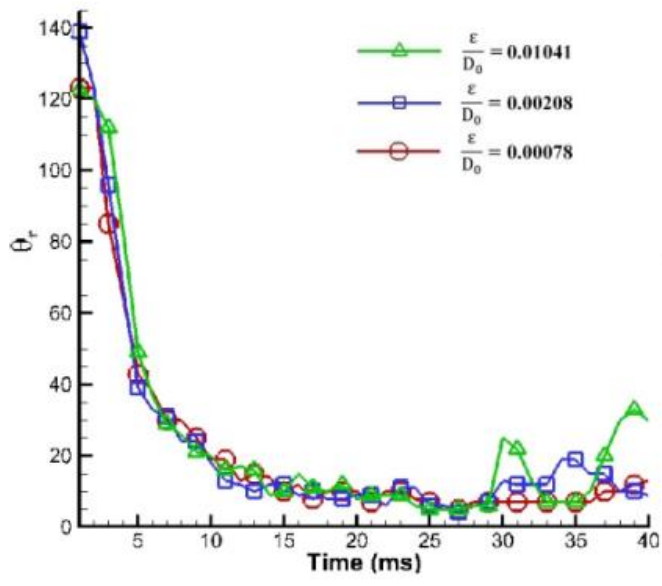

a)

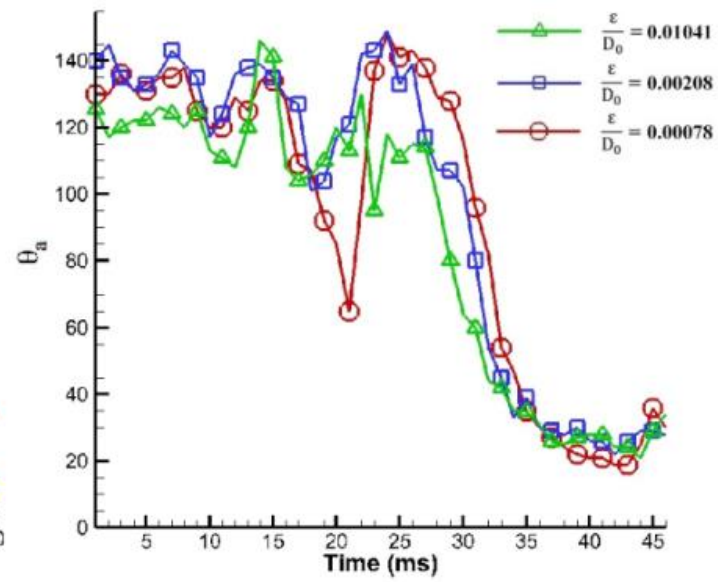

b)

Figure 13. The contact angle variation for inclined impact at $30^{\circ}$ surface angle on stainlesssteel surfaces with different roughness a) $\theta_{r}$ b) $\theta_{a}$ 


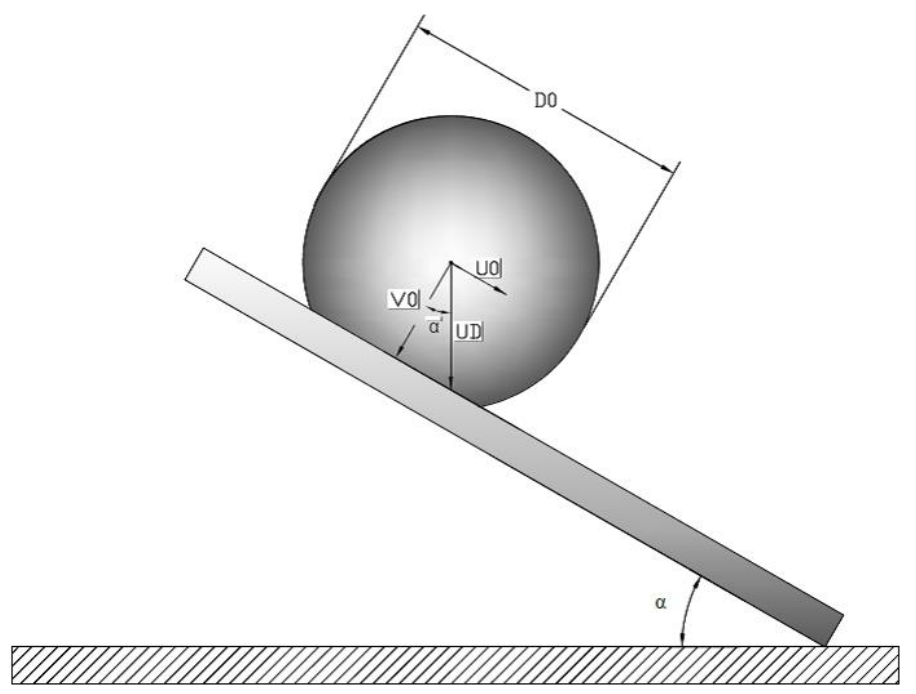

Figure 14. Model of droplet spreading in inclined impact

\section{Empirical Formula of Predicting the Maximum Spread Factor of the Inclined Droplet Impact}

Predicting the maximum spread factor of droplets impacting on inclined surfaces like Figure 14 can be obtained by applying energy conservation equation for a spreading droplet similar to the procedure introduced by Pasandideh-Fard et al. [33]. Several such models are available in the literature [40].

It is assumed that droplet is spherical before impact. Therefore, the kinetic energy $\left(K E_{1}\right)$ and surface energy $\left(S E_{1}\right)$ of the droplet can be expressed as follow [43]:

$$
\begin{gathered}
K E_{1}=\left(\frac{1}{2} \rho U_{D}^{2}\right)\left(\frac{\pi}{6} D_{o}^{3}\right) \\
S E_{1}=\pi D_{0}^{2} \gamma
\end{gathered}
$$

As mentioned in previous sections, the impacting droplet spreads radially until reaches its maximum extension diameter. At this moment, the kinetic energy is equal to zero and the surface energy $\left(S E_{2}\right)$ is as follow [43]:

$$
S E_{2}=\pi \gamma D_{\max } h_{t}+\frac{\pi}{4} D_{\max }^{2} \gamma\left(1-\cos \left(\theta_{o}\right)\right)
$$

The total work done against viscous forces and irreversibly during deformation of the droplet is given by [21]:

$$
W=\int_{0}^{t_{c}} \int_{\Omega} \phi d \Omega d t \approx \phi \Omega t_{c}
$$

where $\Omega, t_{c}$ and $\phi$ are the volume of viscous fluid, the time taken for the droplet to spread, and the viscous dissipation function, respectively. The magnitude of $\phi$ is estimated by[21]:

$$
\phi=\mu\left(\frac{V_{o}}{L}\right)^{2}
$$

where $\mu$ and $L$ are respectively the liquid viscosity and characteristic length in the $Y$ direction. In this model, the boundary layer is chosen as the characteristic length. The boundary layer thickness is given by [33]: 


$$
\delta=2 \frac{D_{o}}{\sqrt{R e}}
$$

The velocity at the edge of the splat during spreading $\left(V_{R}+U_{o}\right)$ can be calculated by applying conservation of mass to the film:

$$
\frac{V_{R}+U_{o}}{V_{o}}=\frac{d^{2}}{4 D h_{t}}
$$

Since the volume of a spherical droplet with diameter $D_{o}$ before impact is equal to the volume of impacting droplet which is a cylinder with height $h_{t}$ (splat thickness) and diameter $D_{\max }$. The $h_{t}$ after impact is [33]:

$$
h_{t}=\frac{2 D_{o}^{3}}{3 D_{\max }^{2}}
$$

$d$ represents the diameter of contact area between impacting droplet and the film. Assuming an average value $d=\frac{D_{o}}{2}$ and combining Eqs. (20) and (21) gives [33]:

$$
\frac{d D}{d t}=2\left(V_{R}+U_{o}\right)=\frac{V_{o} d^{2}}{2 D h_{t}}
$$

The size of splat diameter over time can be determined by Integrating Eq. (22) [33]:

$$
\frac{D}{D_{\max }}=\sqrt{\frac{3}{8} \frac{V_{o}}{D_{o}} t}
$$

The amount of energy dissipated by the viscosity can be calculated by substituting Eqs. (18) and (19) in Eq. (17), assuming that $L=\delta, t_{c}=\frac{8 D_{o}}{3 V_{o}}$ and $\Omega=\frac{\pi D_{\max }^{2} \delta}{4}$ [33]:

$$
W=\frac{1}{3} \pi \rho V_{o}^{2} D_{\max }^{2} D_{o} \frac{1}{\sqrt{R e}}
$$

Using the energy conservation condition $\left(K E_{1}+S E_{1}=S E_{2}+W\right)$ :

where $\sec (\alpha)=\frac{1}{\cos (\alpha)}$

$$
\left(\sec ^{2}(\alpha) W e+12\right) \xi_{\max }=8+\xi_{\max }^{3}\left[3\left(1-\cos \left(\theta_{o}\right)\right)+4 \frac{W e}{\sqrt{R e}}\right]
$$

Equation (25) is a polynomial function of degree 3 . As $\xi_{\max }>1$, the root which satisfies this condition is the answer of above equation. Eq. (25) is only valid when the splashing does not occur, as the maximum extension diameter cannot be determined whenever droplet splashes. For validation of the model, analytical results from Eq. (25) is compared with experimental measurements for the stainless-steel surfaces at 15 and $30^{\circ}$ incline angles (Figure 15 and Table 4). As can be seen in Figure 15, there is a good agreement between the predictions and experimental results; the average discrepancy between the measured and calculated maximum spread factor for the surfaces at 15 and $30^{\circ}$ incline angles are $3.05 \%$ and $5.46 \%$, respectively. The results also show that higher surface roughness leads to lower contact angle. Rough surface dissipates energy more in deforming the droplet stage which means droplet has lower surface energy when impacts on Rough surface. Since there is a direct relationship between droplet surface energy and contact angle, droplet contact angle in Rough surface is smaller compared to surfaces with lower roughness. 


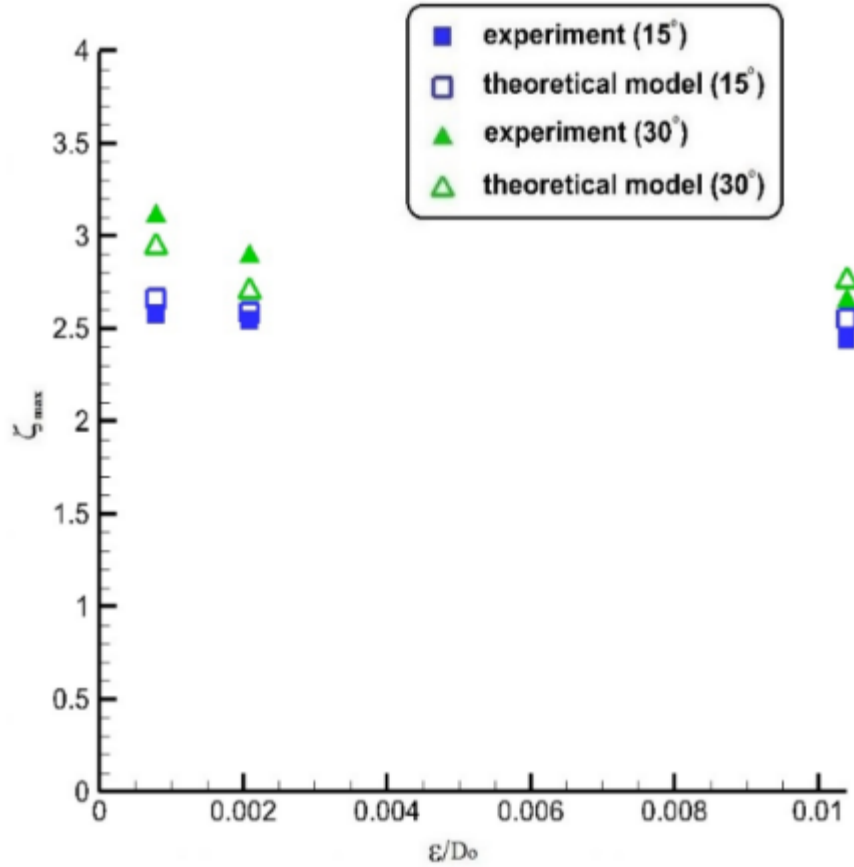

Figure 15. Comparison of maximum spread factor of theoretical model with those of experiments for the inclined droplet impact

Table 4. Comparison of maximum spread factor of theoretical model with those of experiments for the inclined droplet impact

\begin{tabular}{ccccccc}
\hline Case & $\boldsymbol{\alpha}$ & $\frac{\boldsymbol{\varepsilon}}{\boldsymbol{D}_{\boldsymbol{o}}}$ & $\boldsymbol{\theta}_{\boldsymbol{o}}$ & $\begin{array}{c}\boldsymbol{\xi}_{\max } \\
\text { (theory) }\end{array}$ & $\begin{array}{c}\boldsymbol{\xi}_{\max } \\
\text { (experiment) }\end{array}$ & $\begin{array}{c}\text { Difference } \\
\text { error (\%) }\end{array}$ \\
\hline 1 & 15 & 0.000782 & 117 & 2.660 & 2.576 & 3.16 \\
2 & 15 & 0.00208 & 126 & 2.586 & 2.545 & 1.61 \\
3 & 15 & 0.01041 & 131 & 2.550 & 2.438 & 4.39 \\
4 & 30 & 0.000782 & 100 & 2.939 & 3.103 & 5.58 \\
5 & 30 & 0.00208 & 122 & 2.697 & 2.886 & 7.00 \\
6 & 30 & 0.01041 & 116 & 2.753 & 2.648 & 3.81 \\
\hline
\end{tabular}

If $W e$ is large enough ( $W e>100)$, the number 8 in Eq.(25) (term which results from the surface energy of droplet at its maximum extension diameter $\left(S E_{2}\right)$ for inclined impact $\left(\pi \gamma D_{\max } h_{t}\right)$ ) will have little effect on $\xi_{\max }$. Therefore, the maximum spread factor for inclined impact can be obtained:

$$
\xi_{\max }=\frac{D_{\max }}{D_{o}}=\sqrt{\frac{\sec ^{2}(\alpha) W e+12}{3\left(1-\cos \left(\theta_{o}\right)\right)+4 \frac{W e}{\sqrt{R e}}}}
$$

The only difference between Eqs. (10) and (26) is coefficient of $W e$; i.e. $\sec ^{2}(\alpha)$ which is effect of incline angle on maximum spread factor in the inclined impact. In Eq. (26), $\alpha$ varies from $0^{\circ}$ to $90^{\circ}$. For $\alpha=0^{\circ}$ (normal impact), Eqs. (10) and (26) are equal and estimation of 
the maximum spread of a droplet on a surface from both equations are the same. For large

$\frac{W e}{\sqrt{R e}}$, the capillary effects can be neglected. If also $W e \gg 12$, Eq. (25) reduces to

$$
\xi_{\max }=0.5 \sec (\alpha) R e^{0.25}
$$

\section{CONCLUSIONS}

A droplet generator was developed, and the impact of water droplet was captured with a single shot photography method by using a high-speed camera. Experiments were performed to study the effect of surface roughness on the droplet impact characterization. The experimental results showed that the droplet impact consists three main stages: the droplet flattening, the finger instability, and the secondary droplet formation. The asymmetric spreading of droplet impacting on inclined surfaces avoided forming the secondary droplet by decreasing the droplet kinetic energy. It was found that the surface roughness effect on the droplet in the early stages after impact was negligible. After 15-20 milliseconds elapsed from the impact, however, a noticeable difference in droplet deformation can be observed between surfaces with different roughness. Nevertheless, contact angles, in general, were not function of surface roughness. For the same Reynolds and Weber numbers, the viscous dissipation was larger in the surface with high roughness which in turn reduced the kinetic energy. Further improvement can be made in future work by consideration the effect of surface roughness on impact outcomes for different Reynolds and Weber numbers. Also, a simple analytical expression of the maximum spread of a droplet on an inclined surface was derived. Although the accuracy of this model is acceptable, it is required to develop a new model for prediction of the maximum spread factor which separately considers the effect of advancing and receding contact angles. 


\section{REFERENCES}

[1] Schneider T, Kreutz J, Chiu DT. The potential impact of droplet microfluidics in biology. Analytical chemistry. 2013; 85: 3476-82.

[2] Gunasekera DH, Kuek S, Hasanaj D, He Y, Tuck C, Croft AK, Wildman RD. Three dimensional ink-jet printing of biomaterials using ionic liquids and co-solvents. Faraday discussions. 2016 Aug 10;190:509-23.

[3] Khavari M, Sun C, Lohse D, Tran T. Fingering patterns during droplet impact on heated surfaces. Soft Matter. 2015; 11: 3298-303.

[4] Hulwan D, Joshi S. Multizone Model Study for DI Diesel Engine Running on DieselEthanol-Biodiesel Blends of High Ethanol Fraction. International Journal of Automotive and Mechanical Engineering. 2018; 15: 5451-67.

[5] Pai S, Sharief A, Kumar S. Influence of Ultra Injection Pressure with Dynamic Injection Timing on CRDI Engine Performance Using Simarouba Biodiesel Blends. International Journal of Automotive and Mechanical Engineering. 2018; 15: 574859.

[6] Keegan MH, Nash D, Stack M. Modelling rain drop impact of offshore wind turbine blades. ASME Turbo Expo 2012: Turbine Technical Conference and Exposition: American Society of Mechanical Engineers, 2012, p. 887-98.

[7] Goharimanesh M, Javid SM, Bazaz SR, Rostami H. Reducing ice accretion using design of experiments based on Taguchi method. Journal of Applied Science and Engineering. 2017 Jun 1;20(2):165-72.

[8] Mostaghimi J, Chandra S. Droplet Impact and Solidification in Plasma Spraying. Handbook of Thermal Science and Engineering. 2018: 2967-3008.

[9] Kumar R, Sahoo A, Mishra P, Das R. Performance of Near Dry Hard Machining Through Pressurised Air Water Mixture Spray Impingement Cooling Environment. International Journal of Automotive and Mechanical Engineering. 2019; 16: 610833.

[10] Lesser M. Analytic solutions of liquid-drop impact problems. Proceedings of the Royal Society of London Series A, Mathematical and Physical Sciences. 1981; 289308.

[11] Josserand C, Thoroddsen ST. Drop impact on a solid surface. Annual Review of Fluid Mechanics. 2016; 48: 365-91.

[12] Worthington AM. On the forms assumed by drops of liquids falling vertically on a horizontal plate. Proceedings of the royal society of London. 1876; 25: 261-72.

[13] Mundo C, Sommerfeld M, Tropea C. Droplet-wall collisions: experimental studies of the deformation and breakup process. International journal of multiphase flow. 1995; 21: 151-73.

[14] Roisman IV, Rioboo R, Tropea C. Normal impact of a liquid drop on a dry surface: model for spreading and receding. Proceedings of the Royal Society of London A: Mathematical, Physical and Engineering Sciences. 2002: 1411-30.

[15] Kim H-Y, Chun J-H. The recoiling of liquid droplets upon collision with solid surfaces. Physics of fluids. 2001; 13: 643-59.

[16] Kendall GE, Rohsenow WM. Heat transfer to impacting drops and post critical heat flux dispersed flow. Cambridge, Mass.: Heat Transfer Laboratory, Dept. of 
Mechanical Engineering, Massachusetts Institute of Technology, 1978, Report No. 85694-100.

[17] Roisman IV. Inertia dominated drop collisions. II. An analytical solution of the Navier-Stokes equations for a spreading viscous film. Physics of Fluids. 2009; 21: 052104.

[18] Stow C, Hadfield M. An experimental investigation of fluid flow resulting from the impact of a water drop with an unyielding dry surface. Proc R Soc Lond A. 1981; 373: 419-41.

[19] Range K, Feuillebois F. Influence of surface roughness on liquid drop impact. Journal of colloid interface science. 1998; 203: 16-30.

[20] Bussmann M, Mostaghimi J, Chandra S. On a three-dimensional volume tracking model of droplet impact. Physics of Fluids. 1999; 11: 1406-17.

[21] Chandra S, Avedisian C. On the collision of a droplet with a solid surface. Proc R Soc Lond A. 1991; 432: 13-41.

[22] Rioboo R, Marengo M, Tropea C. Time evolution of liquid drop impact onto solid, dry surfaces. Experiments in fluids. 2002; 33: 112-24.

[23] Fujimoto H, Takuda H. Entrapment of air at 45 oblique collision of a water drop with a smooth solid surface at room temperature. International journal of heat mass transfer. 2004; 47: 3301-5.

[24] Li E, Thoroddsen ST. Time-resolved imaging of a compressible air disc under a drop impacting on a solid surface. Journal of Fluid Mechanics. 2015; 780: 636-48.

[25] Li R, Ashgriz N, Chandra S, Andrews JR, Drappel S. Coalescence of two droplets impacting a solid surface. Experiments in Fluids. 2010; 48: 1025-35.

[26] Kolinski JM, Rubinstein SM, Mandre S, Brenner MP, Weitz DA, Mahadevan L. Skating on a film of air: drops impacting on a surface. Physical review letters. 2012; 108: 074503.

[27] Bouwhuis W, van der Veen RC, Tran T, Keij DL, Winkels KG, Peters IR, van der Meer D, Sun C, Snoeijer JH, Lohse D. Maximal air bubble entrainment at liquid-drop impact. Physical review letters. 2012 Dec 26;109(26):264501.

[28] Gier S, Dorbolo S, Terwagne D, Vandewalle N, Wagner C. Bouncing of polymeric droplets on liquid interfaces. Physical Review E. 2012; 86: 066314.

[29] An SM, Lee SY. Maximum spreading of a shear-thinning liquid drop impacting on dry solid surfaces. Experimental Thermal Fluid Science. 2012; 38: 140-8.

[30] Hai-Bao H, Su-He H, Li-Bin C. Droplet impact on regular micro-grooved surfaces. Chinese physics B. 2013; 22: 084702.

[31] Diaz AJ, Ortega A. Gas-assisted droplet impact on a solid surface. Journal of Fluids Engineering. 2016; 138: 081104.

[32] Zohrabi Chakaneh J, Pishbin SJ, Sheikhi Lotfabadi A, Passandideh-Fard M. Experimental and Numerical Characterization of Drop Impact on a Hydrophobic Cylinder. Journal of Fluids Engineering 2019; In Press.

[33] Pasandideh-Fard M, Qiao Y, Chandra S, Mostaghimi J. Capillary effects during droplet impact on a solid surface. Physics of fluids. 1996; 8: 650-9.

[34] Sardarabadi M, Passandideh-Fard M, Heris SZ. Experimental investigation of the effects of silica/water nanofluid on PV/T (photovoltaic thermal units). Energy. 2014; 66: 264-72. 
[35] Castrup H. Error Distribution Variances and Other Statistics. Integrated Sciences Group. 2009; 1: 15.

[36] Ravi V, Jog MA, Manglik RM. Effects of pseudoplasticity on spread and recoil dynamics of aqueous polymeric solution droplets on solid surfaces. Interfacial Phenomena Heat Transfer. 2013; 1.

[37] Hulse-Smith L, Mehdizadeh NZ, Chandra S. Deducing drop size and impact velocity from circular bloodstains. Journal of Forensic Science. 2005; 50: JFS2003224-10.

[38] Minemawari H, Yamada T, Matsui H, Tsutsumi JY, Haas S, Chiba R, Kumai R, Hasegawa T. Inkjet printing of single-crystal films. Nature. 2011 Jul;475(7356):364.

[39] Attinger D, Moore C, Donaldson A, Jafari A, Stone HA. Fluid dynamics topics in bloodstain pattern analysis: comparative review and research opportunities. Forensic science international. 2013; 231: 375-96.

[40] Bennett T, Poulikakos D. Splat-quench solidification: estimating the maximum spreading of a droplet impacting a solid surface. Journal of Materials Science. 1993; 28: 963-70.

[41] Bertozzi AL, Brenner MP. Linear stability and transient growth in driven contact lines. Physics of Fluids. 1997; 9: 530-9.

[42] Tadmor R. Line energy and the relation between advancing, receding, and young contact angles. Langmuir. 2004; 20: 7659-64.

[43] Carey VP. Liquid-vapor phase-change phenomena. 1992. 